\title{
Respiratory Tract Infections: Sinusitis, Bronchitis, and Pneumonia
}

\author{
Benjamin A. Miko, Marcus R. Pereira, and Amar Safdar
}

\section{Introduction}

Upper and lower respiratory tract infections are among the most common infectious processes among solid organ and hematopoietic stem cell transplant (HSCT) recipients [1]. As with most infectious syndromes outlined in this text, the increased risk of respiratory tract disease encountered in transplant recipients relates in part to defects in innate and adaptive immunity. Within this population, however, the incidence and microbiological etiology of respiratory infections are quite variable. This is due in part to the use of heterogeneous immunosuppressive regimens and differing prophylactic antibiotic strategies. In addition to diminished host immunity, anatomic breaches in upper and lower respiratory tract defenses may increase the susceptibility of immunocompromised hosts to a variety of common and opportunistic pathogens. Such anatomical considerations are especially important in patients who have undergone thoracic surgery for lung and heart transplantation and individuals who have required prolonged mechanical ventilation. Medical devices such as nasogastric and endotracheal tubes hinder coordinated glottic movement and mucociliary function and act as conduits for the introduction of pathogenic organisms to the respiratory tract [2].

Several immune deficits can affect transplant recipients and consequently increase the risk of upper and lower respiratory tract infections. These include:

\section{B. A. Miko}

Columbia University Medical Center, Division of Infectious Diseases, Department of Medicine, New York, NY, USA e-mail: bm2266@columbia.edu

\section{R. Pereira}

Department of Medicine - Infectious Diseases, Columbia University Medical Center, New York, NY, USA

e-mail: mp2323@cumc.columbia.edu

A. Safdar $(\square)$

Clinical Associate Professor of Medicine, Texas Tech University

Health Sciences Center El Paso, Paul L. Foster School of

Medicine, El Paso, TX, USA

e-mail: amar.safdar@cidimmunology.com
1. Neutropenia: Commonly associated with medication toxicities, chemotherapy, hematological malignancies, and viral infections, deficits in neutrophil count and function can increase the risk of infection with staphylococci, streptococci, Gram-negative bacilli (GNB), and fungi $[3,4]$.

2. T lymphocyte deficiency: Caused by many transplant immunosuppressive medications that include calcineurin inhibitors, mTOR inhibitors, glucocorticoids. T lymphocyte deficiency is also encountered in the setting of HIV infection, lymphoma, leukemia, and infection/reactivation of various herpesviruses, especially Cytomegalovirus (CMV). Patients with cellular immune dysfunction are at increased risk of respiratory infection due to intracellular organisms such as Listeria monocytogenes, Salmonella spp., Legionella spp., and Toxoplasma gondii, mycobacteria, fungi-like Cryptococcus spp., Histoplasma capsulatum, and Pneumocystis jirovecii, as well as CMV, Human herpesvirus 6 (HHV-6), varicella-zoster virus (VZV), and respiratory viruses [5-7].

3. B lymphocyte deficiency: Associated with various transplant/oncology medications like azathioprine, mycophenolate, rituximab, and glucocorticoids and hematological malignancies such as leukemia, multiple myeloma, and other causes of humoral immune defects increases the risk of infection with encapsulated bacteria including $S$. pneumoniae, $H$. influenzae, and N. meningitidis.

4. Asplenia and hypocomplementemia: Similarly increase the risk of infection with encapsulated bacteria as well as Capnocytophaga.

Because such immune defects are often mixed, careful attention to clinical and radiographic features as well as recognition of nosocomial versus community sources of infection are critical to making a correct diagnosis and initiating empiric antimicrobial therapy [1]. In both solid organ transplant and HSCT recipients, delays in appropriate antimicrobial therapy may increase the risk of secondary complications and infection-associated deaths, especially in those with 
severe immunosuppression. Therefore, it is common practice to initiate empiric or preemptive antimicrobial therapy in patients for whom the suspicion of infection is high.

This chapter reviews the common respiratory tract infections affecting transplant recipients including sinusitis, bronchitis, tracheobronchitis, and pneumonia. Particular attention is directed to epidemiological risk factors including healthcare exposure, clinical presentation, diagnosis, and common microbiological etiologies. Unique causes of opportunistic pneumonias are reviewed in the final section of this chapter.

\section{Sinusitis}

Sinus infections are a common occurrence in transplant recipients, affecting between $4 \%$ and $31 \%$ of HSCT recipients $[8,9]$ and probably a similar percent of solid organ transplant recipients [10]. Of note, transplant patients with underlying cystic fibrosis $(\mathrm{CF})$ are at extremely high risk for sinusitis with between $90 \%$ and $100 \%$ of individuals with $\mathrm{CF}$ showing pan-sinusitis on imaging by 8 months of age [11]. In HSCT recipients, risk factors for developing severe rhinosinusitis include graft-versus-host disease (GVHD) [12, 13]. The determinants of sinusitis in solid organ transplant recipients are less clearly defined, possibly due to the heterogeneous disease processes prompting transplantation. Unlike non-immunosuppressed individuals, no studies have linked tobacco, allergy, asthma, and low IgG levels to sinusitis in transplant recipients [13].

In general, sinusitis can be classified temporally as acute ( $<4$ weeks), subacute (4-12 weeks), or chronic ( $>12$ weeks) and in terms of severity as invasive or noninvasive. Typical signs and symptoms include nasal congestion, focal sinus pressure or pain, nasal discharge, and reduced sense of smell although, as is generally the case, transplant recipients can often have a muted clinical picture. Among HSCT recipients, nasal congestion and cough are the most common symptoms of acute sinusitis $(80 \%$ and $61 \%$, respectively) [8]. The nonspecific nature of these symptoms may ultimately lead to delays in diagnosis resulting in poorer clinical outcomes of invasive infections.

The diagnosis of acute sinusitis is often based entirely on clinical presentation in the immunocompetent patients, whereas in transplant recipients, a high degree of suspision due to dampened clinical signs and symptoms should be accompanied by an appropriate diagnostic investigation that often includes CT or MRI scan of the face and paranasal sinuses. In most cases, cultures of nasal discharge are not helpful; a nasal wash for bacterial cultures and a PCR panel that assesses a number of common and uncommon respiratory viruses may considerably improve diagnostic yeild. When imaging is obtained, fluid levels are found in $86 \%$ of cases [6]. Although a wide variety of organisms can cause acute sinusitis, the vast majority of infections are due to viral pathogens. These include rhinovirus, respiratory syncytial virus (RSV), adenovirus, coronavirus, influenza, parainfluenza, and even CMV [14-16]. Viral infections can be short-lived but viral shedding can be prolonged among immunocompromised patients. Bacterial sinus infections are much less common and rarely occur with less than 7 days of symptoms [17]. Common bacterial causes of acute bacterial sinusitis include $S$. pneumoniae, Haemophilus influenzae, Moraxella catarrhalis, S. aureus, and anaerobes. Pseudomonas can be a prominent sinus pathogen in cystic fibrosis patients both before and after lung transplantation [18, 19]. While most cases of acute bacterial sinusitis are caused by one predominant organism, up to one quarter have two distinct pathogens present in high concentration [20]. It is important to note, however, that in routine clinical practice, the majority of patients with acute sinusitis do not have a bacterial pathogen isolated.

In the immunocompetent patients treatment of acute sinusitis is usually supportive except in cases where symptoms persist for greater than 7 days, at which point empiric antimicrobial therapy may be warranted. In severely immunocompormised transplant patients with or without neutropenia, approach has been to start treatment early when bacterial sinus infection is suspected. Complications of acute sinusitis include facial cellulitis, preseptal periorbital cellulitis, brain abscess, cavernous sinus thrombosis, and orbital invasion that may result in sight and life threatening orbital (post-septal) cellulitis [21]. Intracraneal extension should be suspected if mental status changes occur or if there are focal neurological signs [22]. CT imaging or MRI scan should be urgently obtained, and otolaryngology evaluation with endoscopy should be sought for both diagnosis (cultures and histology) and therapeutic drainage. Empiric antimicrobials should be initiated while this diagnostic evaluation is pending. In lung transplant recipients with underlying cystic fibrosis, sinus surgery can decrease recurrence of Pseudomonas-related sinus infections [18].

Invasive fungal sinus infections are associated with increased morbidity and mortality that may range from $50 \%$ and may beupto as high as $90 \%$ in patients undergoing solid organ allograft and allogenic hematopoietic stem cell transplantation [23]. Bony erosions can be a sign of invasive disease. There is a correlation with chronicity and invasiveness. Invasive sinus disease is often fungal although bacterial causes such as Pseudomonas and S. aureus are not uncommon. In transplant recipients, Candida and Aspergillus species remain the most common fungal organisms to cause sinusitis, resulting in invasive and noninvasive sinus disease [24]. Rhizopus is also an important organism associated with rapidly progressive infection with high mortality rate [21]. Even rarer fungal etiologies include Scopulariopsis [25], Fusarium [26, 27], Trichoderma [28], Scedosporium [29], and Pseudallescheria [30]. Acanthamoeba is a rare but well-described parasitic cause of invasive rhinosinusitis in immunosuppressed patients with a strong predilection for intracraneal involvement, in such patients brain infection carries high fatality $[31,32]$. 


\section{Bronchitis and Tracheobronchitis}

The terms tracheobronchitis and acute bronchitis are frequently used interchangeably, with acute bronchitis having a preferential use in the literature with the exception of aspergillosis and ventilator-associated tracheobronchial infections [33-35]. As such, both are generally defined as a self-limited inflammation of the large airways of the lung, or bronchi, due to an infection. As opposed to pneumonia, the lower airways are not involved, and chest imaging is generally normal. Patients typically present with a cough lasting more than 5 days, an important factor distinguishing it from mild upper respiratory infection (URI) [36]. Symptoms usually persist for 10-20 days but may extend for more than 4 weeks. Most patients report purulent sputum, indicating sloughing of tracheobronchial epithelium and inflammation. As opposed to bronchiolitis, acute bronchitis does not usually present with progressive cough, wheezing, tachypnea, respiratory distress, and hypoxemia. Chronic bronchitis, on the other hand, entails continuing symptoms on most days of the month for at least 3 months of the year during two consecutive years.

Many studies have shown that community-acquired viruses are by far the most common cause of acute bronchitis [37-40]. These include influenza $\mathrm{A}$ and $\mathrm{B}$, adenovirus, rhinovirus, coronavirus, parainfluenza, RSV, and human metapneumovirus [37-40]. Bacteria, particularly atypical organisms, can also cause acute bronchitis; commonly implicated pathogens include Bordetella pertussis, Chlamydophila pneumoniae, and Mycoplasma pneumonia. Among immunocompromised patients, the causative agents of acute bronchitis are largely similar to the general population [41-43]. Opportunistic pathogens can also cause tracheobronchial infections, particularly among lung transplant and allogeneic HSCT recipients. The most important of these include invasive fungal infections, especially Aspergillus [35, 44, 45].

Acute bronchitis affects about $5 \%$ of adults annually, with the majority of infections occurring during the fall and winter [46]. The incidence of upper respiratory tract infections among solid organ transplant and HSCT recipients appears to be similar to that of the general population [43, 47-49]. Despite similar epidemiology, immunocompromised patients often suffer from prolonged periods viral shedding and progress to pneumonia more frequently [50]. Higher rates of airflow obstruction and increased mortality are also key differences $[50,51]$. Respiratory viral infections immediately prior to bone marrow transplant are associated with decreased survival [52]. Among lung transplant recipients, respiratory viral infection, particularly RSV, can be associated with progression of chronic rejection and bronchiolitis obliterans syndrome (BOS) on histopathologic examination $[41,47,48,53]$. Patients undergoing allogeneic hematopietic stem cell transplantation similarly are at risk for BOS that potentially may have been triggered after an episode of respiratory viral infection [54].
The major procedures employed for diagnosis of upper respiratory tract infections include multiplex PCR platforms and sputum cultures. When a fungal etiology is suspected, bronchoscopy is often performed to establish microbiological diagnosis. Among lung transplant recipients, examination of the bronchial anastomosis is essential to ascertain its integrity and the presence of necrosis.

Treatment for acute bronchitis depends on the causative infectious agent [51]. For the viral organisms without an established therapy, supportive care and close monitoring is recommended. Please refer to each specific pathogen for further information on treatment.

\section{Pneumonia}

Guidelines put forth by the Infectious Diseases Society of American (IDSA) and American Thoracic Society (ATS) suggest that the diagnosis of pneumonia requires a constellation of suggestive clinical features such as fever, purulent sputum, leukocytosis, and decline in oxygen saturation, and a demonstrable infiltrate on chest radiograph or other imaging technique with or without supporting microbiological data $[55,56]$. As the common clinical and radiographic manifestations of pneumonia may be absent or attenuated in immunosuppressed patients due to impaired inflammatory responses [1], the diagnosis of pneumonia may be difficult to establish in solid allograft transplant and both autologous and allogeneic HSCT recipients. Adding to this diagnostic challenge is the frequent colonization of the upper airway with microorganisms that do not contribute to lung disease, rendering the microbiological diagnosis of pneumonia by conventional culture techniques difficult. Conversely, sterile respiratory tract cultures do not exclude an infectious etiology, particularly in the setting of recent exposure to broadspectrum antibiotics. The common use of prophylactic antimicrobials in transplant recipients may contribute to suppression of culture results while increasing the risk of infections that are resistant to commonly used antimicrobial agents given for infection prophylaxis such as fluoroquinolones, trimethoprim-sulfamethoxazole, triazole-based antifungals, and valacyclovir or valganciclovir [57-59].

As in the general population, pneumonias occurring in transplant recipients are traditionally defined based on the setting in which they are acquired, i.e., communityacquired vs. hospital-acquired. Until 2016, the IDSA and ATS designated a third category, healthcare-associated pneumonia (HCAP), to delineate patients with significant exposure to nursing homes, dialysis centers, and outpatient clinics. While this was intended to highlight this group's increased risk of multi-drug resistant pathogens, several studies have shown HCAP to be caused by organisms similar to those causing community-acquired pneumonia (CAP) [60-62]. Given that, the HCAP designation was removed from the most-recent IDSA/ATS guidelines for 
the management of hospital-acquired pneumonia (HAP) and ventilator-associated pneumonia (VAP) [56].

\section{Community-Acquired Pneumonia}

CAP, as defined by IDSA and ATS, refers to the radiographic and clinical development of pneumonia in the community setting, distinguishing it from HAP, as outlined below $[55,56]$. The distinction of CAP from nosocomial pneumonia remains important as it allows for prediction of likely pathogens and permits prognostic estimations based on epidemiologic descriptions of the underlying cause. Consequently, this distinction provides a framework for decisions regarding the appropriate diagnostic evaluation and empiric antimicrobial therapy.

The etiologic spectrum of bacterial pathogens causing CAP among transplant recipients with mild-to-moderate immunosuppression is similar to that of patients without a history of transplantation. However, a clinically insignificant microbial inoculum in the general population may cause severe infection among patients with underlying immunosuppression. S. pneumoniae remains the most commonly identified pathogen and the most frequent cause of lethal CAP [63]. S. aureus, nontypeable Haemophilus influenzae, Pseudomonas spp., and other GNB may also cause lifethreatening CAP. Recently, other non-lactose fermenting (NF)-GNB such as Stenotrophomonas, Burkholderia, Chryseobacterium, Achromobacter, and Alcaligenes species have been increasingly recognized as etiologic agents in both CAP and nosocomial infections [64]. S. pyogenes, Neisseria meningitidis, and Moraxella catarrhalis also cause CAP less frequently. The incidence of CAP associated with the atypical pathogens such as Mycoplasma pneumoniae, Chlamydia pneumoniae, and Legionella spp. varies widely with patient age, seasonal variation, and geographic location. Viral pneumonias, most commonly caused by influenza, parainfluenza, and adenovirus, are also sources of CAP, which may be severe in the setting of transplantation. It is important to note that lower respiratory tract infections may be due to a mixed population of viruses, bacteria, and fungi in solid organ transplant and HSCT recipients [65-70].

The microbiological diagnosis of CAP is based upon recovery of a likely pathogen from an otherwise sterile source like blood, urine, pleural fluid, isolation of a noncommensal organism in respiratory secretions, or positive results of selected serological tests. Although the utility of Gram staining and culture of expectorated sputum in the diagnosis of pneumonia has been debated for years, carefully procured sputum specimens with cytologic confirmation of a lower respiratory source appear to be diagnostically useful, particularly if they are obtained before the initiation of antimicrobial therapy. Timely establishment of an accurate diagnosis contributes to a successful outcome, although treatment should not be withheld while diagnostic interventions are underway. Antimicrobial selection should be based upon the probable infecting organism(s), the severity of the patient's pneumonia, the patient's underlying immune status, and the presence or absence of comorbid conditions [71, 72].

\section{Hospital-Acquired Pneumonia}

Lower respiratory tract infections that occur more than $48 \mathrm{~h}$ after hospital admission in patients without antecedent clinical symptoms or radiographic findings suggestive of pneumonia are referred to as HAP. The etiological spectrum of microbial pathogens causing HAP among low-risk transplant recipients with no recent antibiotic exposure is similar as that seen in the general population. H. influenzae, S. pneumoniae, S. aureus, and Enterobacteriaceae are frequently encountered. Methicillin-resistant S. aureus (MRSA) may cause severe HAP, especially among patients with prior MRSA colonization, antibiotic exposure, advanced age, and/or prolonged ventilatory support [73]. Protracted mechanical ventilation and recent antibiotic administration are also associated with increased rates of HAP caused by $P$. aeruginosa, Acinetobacter baumannii complex, Enterobacter spp., and emerging strains of MDR NF-GNB such as $S$. maltophilia, Burkholderia cepacia complex, and Alcaligenes (Achromobacter) species, which may be difficult to treat. Mortality rates associated with HAP due to MRSA or $P$. aeruginosa are disproportionately higher than those caused by other nosocomial bacterial pathogens [74].

Polymicrobial isolates and MDR pathogens are more common among patients with HAP, particularly when it occurs as a late complication during hospitalization. Because of the frequency with which multiple organisms are identified on a single respiratory sample, recent evidence-based guidelines advocate the use of quantitative or semiquantitative lower respiratory tract cultures obtained either bronchoscopically or noninvasively as part of the initial evaluation of the patients with suspected HAP or VAP [75].

Empiric antibiotic selections for HAP that develop within 7 days of admission should target $S$. pneumoniae, S. aureus including MRSA, Streptococcus spp., H. influenzae, and Enterobacteriaceae. Patients with late HAP occurring $>1$ week after hospitalization should receive empiric antimicrobial therapy that includes coverage for MDR-GNB. The scope of alternative antimicrobial choices in patients with refractory or slow-to-respond HAP or VAP should be based on institution-dependent susceptibility profiles. If an institution's incidence of MRSA pneumonia is low and respiratory cultures are unavailable or unrevealing, screening for MRSA nasal colonization may be useful in guiding therapy [76]. As the absence of MRSA nasal colonization has a high negative predictive value for MRSA pneumonia, coverage for that organism can often be discontinued early in the treatment course if screening swabs are negative [76]. 


\section{Pneumonias Caused by Aspiration and Bronchial Obstruction}

Aspiration of orogastric contents and mechanical obstruction of the airways may create a favorable milieu for pneumonia caused by microaerophilic or anaerobic bacteria like Peptostreptococcus spp. A variety of factors, such as abnormal swallow function, altered cough reflex, impaired mucociliary clearance, altered mental status, the use of sedating medications, chemotherapyinduced mucositis, supine positioning, gastroparesis, mechanical ventilation, and nasogastric tube feeding, all contribute to the increased predilection for aspiration in patients with histories of transplantation or malignancy [77-79]. Pneumonia associated with large-volume aspiration of gastric contents typically occurs as a late finding. The acidic gastric contents act as a poor medium for bacterial growth. Thus, the initial clinical syndrome following aspiration of gastric contents arises from the direct caustic effect of the acidic aspirate on the cells of the alveolarcapillary interface, i.e., chemical pneumonitis. A true bacterial pneumonia, if it occurs, is consequently a superimposed process. Aspiration of oral contents, by contrast, results from inhalation of nonsterile oropharyngeal material. The clinical presentation is often insidious, and the diagnosis is commonly inferred based on a compatible patient risk profile coupled with radiographic evidence of pneumonia.

Chest radiographs may show focal abnormalities that correlate with the patient's position at the time of aspiration. For example, aspiration that occurs while the patient is in the upright position typically localizes to the basilar segments of the lower lobes, whereas the superior segments of the lower lobes and posterior segments of the upper lobes are more frequently affected following aspiration that occurs in the supine position. The major pathogens underlying nosocomial versus community-acquired aspiration pneumonias differ although a microbiologic diagnosis may not be established due to the limited yield of conventional anaerobic cultures [78, 80-82]. If necessary, such cultures may be best obtained bronchoscopically using a protected strategy.

The management of patients with significant lung injury associated with the aspiration of gastric contents includes aggressive supportive care. Upper airway suctioning, pulmonary toilet, and, if necessary, positive pressure ventilation comprise the mainstays of therapy. There is no clearly established role for corticosteroids in this setting, though the practice of prescribing moderate- to high-dose prednisolone is not uncommon. Early and aggressive antimicrobial therapy is recommended for patients with pneumonia secondary to aspiration of oropharyngeal contents. Antimicrobial selections should be tailored to the immune status of the patient and setting in which the aspiration occurred, i.e., community vs. healthcare environment but in general should be broad in spectrum and target Gramnegative organisms and oral anaerobes. Anaerobic coverage may be particularly important in patients with periodontal disease, putrid sputum, or evidence of necrotizing pneumonia [78].

\section{Other Sources of Pneumonia}

Transplant recipients with altered pulmonary anatomy specifically lung transplant recipients with bronchial anastomotic strictures may be at risk for obstruction of the airways, atelectasis, and postobstructive pneumonia. The associated pneumonias tend to be polymicrobial in nature including GNB, staphylococci, and anaerobes and may require relief of the obstruction to achieve adequate antimicrobial effects, even if appropriate antibiotics are selected. This is often most rapidly achieved through interventional bronchoscopic techniques such as bronchial dilation with or without stent placement.

The lungs may also become infected via septic emboli arising from suppurative endovascular bacterial and, less commonly, fungal infections. Infected intravascular septic deep venous thrombi are increasingly recognized as a potential source of infection in immunosuppressed patients. The radiographic pattern in these patients is distinctive and includes multicentric, pleomorphic lung nodules with asymmetric, relatively small, thick-walled cavities.

\section{Specific Pathogens}

Opportunistic organisms commonly implicated in transplantrelated respiratory tract infections are outlined below.

\section{Nocardia and Actinomycosis}

Over 30 species of Nocardia have been associated with human disease [83]. Nocardia asteroides complex, including $N$. asteroides sensu stricto and $N$. farcinica, accounts for nearly $90 \%$ of Nocardia infections, both in cancer patients and the general population. Risk factors for Nocardia pneumonia include profound deficiencies in cellular immunity, prolonged use of high-dose systemic corticosteroids, especially in the treatment of chronic lung diseases [84], and the presence of GVHD. Solid organ transplant recipients are also at particular risk for Nocardia infections although this varies based upon the organ transplanted. One review demonstrated infection rates of 3.5\%, $2.5 \%, 1.3 \%, 0.2 \%$, and $0.1 \%$ among recipients of the lungs, hearts, intestines, livers, and kidneys, respectively [85]. Nocardia infections commonly occur within the 1st year of transplantation although early $(<1$ month $)$ and late ( $>2$ years) infections have also been reported [85-87]. Infection with the organism should be considered if nodular pulmonary infiltrates are seen, although reticulonodular or diffuse infiltrates are occasionally described. Solitary nodules associated with irregular, thick-walled cavities that mimic invasive pulmonary aspergillosis, histoplasmosis, necrotizing cancer, or chronic bacterial lung abscess have also been associated with Nocardia infection. 
Indolent Nocardia pneumonia may be clinically indistinguishable from other actinomycetes infections and from pneumonias caused by pulmonary eumycetes. Severely immunosuppressed cancer patients with refractory leukemia or prior allogenic HSCT may present with rapidly progressive multifocal nocardiosis. Spontaneous pneumothorax and hemoptysis are also recognized presentations of Nocardia infection among immunocompromised patients. Concomitant brain involvement is not uncommon, and preemptive evaluation is recommended to diagnose asymptomatic brain abscess in patients with pulmonary Nocardia infection. Trimethoprimsulfamethoxazole $(10-12 \mathrm{mg} / \mathrm{kg}$ daily) is effective against many Nocardia species. Retrospective studies suggest that clinical outcomes are improved when appropriate therapy is given for an extended period of time (6-12 months) [88]. Despite aggressive antimicrobial therapy, pulmonary nocardiosis carries a high mortality in immunosuppressed individuals [84]. Pulmonary actinomycosis typically presents in a very similar manner to nocardiosis, although it is classically associated with invasion across tissue plans. As such, pulmonary infection may involve the adjoining pleura and subsequently erode through the chest wall. Isolation of Actinomycetes from the respiratory tract should be evaluated critically as their presence may represent oropharyngeal contamination.

\section{Tuberculosis}

Mycobacterium tuberculosis is a rare cause of pulmonary infections in the developed world but is important to consider in severely immunosuppressed patients, especially foreign-born individuals or patients undergoing allograft solid organ or stem cell translantation in the developing countries where tuberculosis is regarded as an endemic disease [89]. Solid organ transplant recipients are estimated to have 20-74 times higher incidence of active tuberculosis than that of the general population [90]. Frequency of disease varies based upon the organ transplanted and the time from transplantation, with twothirds of cases occurring within 1 year of transplantation [91].

A broad range of clinical manifestations may be possible with tuberculosis infection. Pulmonary tuberculosis may present as an insidious pneumonia that is difficult to distinguish from Actinomycetes and eumycetes infection. Patients with impaired T-cell response may develop rapidly progressive tuberculosis that follows the course of a virulent bacterial infection. Systemic corticosteroid therapy is an independent predictor of both tuberculosis reactivation and suboptimal response to combination antimicrobial therapy. Hence, once the diagnosis of tuberculosis is established, every effort should be made to discontinue steroid therapy if not indicated for a specific syndrome [89]. Just as HIV-infected patients may develop clinical worsening of tuberculosis pneumonia when initiating antiretroviral therapy such as immune reconstitution inflammatory syndrome, tuberculosis-related lung disease in solid organ transplant or HSCT recipients may infrequently worsen as immune function recovers following temporary discontinuation or partial withdrawal of antirejection or antiGVHD therapy. Nonetheless, minimizing immunosuppression may be helpful in clearing such infections.

\section{Nontuberculous Mycobacteria}

Nontuberculous mycobacteria (NTM) are ubiquitous in the environment and generally cause infection only in hosts with specific anatomical or immunological defects [92]. Transplant recipients are at particular risk due to their impaired cell-mediated immunity. Among patients with structural lung disease particularly those before and after lung transplantation, this risk is further compounded by anatomical abnormalities. Epidemiological data are somewhat lacking for these pathogens as NTM infections are not reportable infections [92]. Incidence rates for NTM pulmonary infections are estimated at $0.24-2.8 \%$ among heart transplant recipients and $0.46-$ $8.0 \%$ among lung transplant recipients [93-95].

Pulmonary NTM infections are classically caused by $M$. avium-intracellulare complex and other slow-growing mycobacteria. These opportunistic pathogens are most frequently associated with chronic, indolent pneumonias. In the United States, the rapidly growing mycobacteria particularly M. abscessus and M. fortuitum have emerged as another important, albeit less frequent cause of NTM lung disease. The diagnosis of pulmonary NTM infections remains a challenge as identification of these organisms in respiratory cultures may result from colonization of the respiratory tract or environmental contamination. Causality is suggested by identification of NTM in sterile lower respiratory tract specimens coupled with corresponding clinical manifestations such as chronic nonproductive cough and exertional dyspnea and a NTM lung disease compatible radiographic presentation. Fever, night sweats, weight loss, pleuritic chest pain, and pleural effusions are also possible but less frequent in the absence of systemic disseminated infection.

Radiographic features of NTM infection include upper lobe predominant nonspecific nodular lesions and small, thinwalled cavities. Chest CT findings demonstrating the characteristic "tree-in-bud" appearance may also be seen in patients with other chronic infections. The so-called Lady Windermere syndrome, characterized by relapsing or refractory pulmonary NTM infection due to slow-growing mycobacteria, may be seen in patients with defects in endogenous interferon-gamma cellular immune response [96]. NTM pulmonary infections are usually insidious, although rapidly progressive disease has been seen in patients with profound defects in helper T-cells. Treatment should include at least two antimicrobial agents to which the Mycobacterium is susceptible. 


\section{Pneumocystis jirovecii}

Similar to NTM, P. jirovecii previously known as $P$. carinii is thought to be ubiquitous in the environment and only causes infection in the setting of impaired immunity. Classically described in HIV-positive individuals with pronounced CD4 lymphocytopenia, Pneumocystis is an important pathogen in solid organ transplant recipients [97]. In most immunosuppressed patients, Pneumocystis pneumonia presents as a slowly progressive infection accompanied by nonproductive cough, exertional dyspnea, and hypoxemia, although an acute, rapidly progressive form has been described. CT evidence of perihilar infiltrates may be mistaken for pneumonitis caused by common acquired viral infections such as RSV, influenza, parainfluenza, or CMV during the early phase of the infection. Bronchoalveolar lavage typically has a high diagnostic yield either through silver staining or through PCR amplification. High-dose trimethoprim-sulfamethoxazole (15-20 mg/kg daily) given for 21 days is the treatment of choice. Adjuvant systemic corticosteroids should be administered to most patients with severe hypoxemia. Oral atovaquone, primaquine plus clindamycin, and parenteral pentamidine may be given to patients who are intolerant of sulfa-containing regimens.

\section{Invasive Fungal Pneumonia}

Invasive pulmonary aspergillosis (IPA) is a relatively common cause of pneumonia in patients undergoing allogeneic HSCT and severely immunosuppressed patients following highrisk solid organ allograft transplantation [98]. Among cancer patients, risk factors for invasive pulmonary aspergillosis include prolonged ( $>1$ week) and severe $(<100$ cells $/ \mu \mathrm{L})$ neutropenia, refractory leukemia, allogeneic HSCT, GVHD, immunosuppressive therapy, and treatment with high-dose systemic corticosteroids $[99,100]$. Among solid organ transplant recipients, risk factors vary based upon the organ transplanted but often include renal failure, reoperation/retransplantation, and CMV infection [101]. Aspergillus fumigatus is most commonly encountered, although non-fumigatus Aspergillus species are increasingly recognized. Similarly, a marked increase in pulmonary invasive fungal infections due to non-Aspergillus molds including Fusarium, Pseudallescheria boydii, and Scedosporium spp. and the dematiaceous (black) molds has also been noted, making the selection of an effective empiric regimen more challenging. The increased incidence of pulmonary mucormycosis may be related to changes in antifungal utilization with a shift away from amphotericin B compounds in favor of mold-active triazole drugs like voriconazole [102]. The effects of newer triazole agents like posaconazole and isavuconazonium on the overall feasibility, efficacy and safety in magagement of fungal pneumonia in transplant recipients is evolving and appears encouraging.
While the clinical symptoms of fungal pneumonia may be similar to those seen in bacterial pneumonia, CT imaging may reveal a highly suggestive "halo sign" during the early course of infection and/or less often observed "crescent sign" that becomes apparent during the later course of IPA. Despite this, in most cases of pulmonary mycosis, the only radiographic findings at the time of presentation are peripheral, pleural-based lung nodules, sometimes with thickwalled regular or irregular cavities [103]. The definitive diagnosis of pulmonary invasive fungal infection requires demonstration of fungal hyphae within the involved lung tissue. Therefore, the clinical diagnosis is often made by inference as high prevalence of thrombocytopenia and coagulopathies in transplant recipients render lung biopsies unsafe. It is important to note that isolation of fungi in respiratory samples may misrepresent the etiology of underlying pulmonary infiltrates as they may reflect environmental contamination or respiratory tract colonization.

The measurement of fungal antigens such as serum galactomannan, bronchoalveolar lavage galactomannan, and serum beta-D-glucan can aid in the detection of invasive pulmonary mycosis. Newer assays, including sequence-based nucleic acid amplification techniques, may further alter the diagnostic strategies used for invasive fungal infection in the future. Therapeutic strategies for these infections are discussed elsewhere.

\section{Viruses}

As previously noted, respiratory viruses including RSV, influenza $\mathrm{A}$ and $\mathrm{B}$, parainfluenza, and adenovirus are common causes of upper respiratory tract infections that may have lower respiratory tract manifestations in immunosuppressed patients. Human metapneumovirus (hMPV) is also recognized as a serious pulmonary pathogen in this population. The spectrum of hMPV disease may range from mild upper respiratory tract infection to serious disseminated infection leading to respiratory failure and encephalitis. Herpesviruses, particularly CMV, are also important causes of pneumonitis in transplant recipients. As invasive CMV disease often affects the transplanted allograft, lung transplant recipients are at particularly high risk of CMV pneumonitis [104]. The virus itself is immunomodulatory in nature, and CMV has been associated with bacterial and fungal superinfections as well as lymphoproliferative disorders [105, 106].

Fever and nonproductive cough are prominent but nonspecific features of viral respiratory tract infection. In patients with extensive lung involvement, dyspnea may appear early in the course of infection. Viral nucleic acids in nasal washes, tracheal aspirates, and bronchial specimens are most frequently used in diagnosing viral respiratory infections through multiplex PCR platforms. Despite that, 
the isolation of CMV especially by PCR amplification from lower respiratory tract secretions may not necessarily indicate CMV lung infection as even in transplant recipients with severe cellular immune defects; intermittent low-level viral replication and shed virus without developing viral lung disease has been well established. Hosts' risk assessment and CMV disease susceptibility evaluation along with radiographic imaging such as non-iv-contrast chest CT scan prove helpful in discerning active viral lung disease versus nondisease associated CMV respiratory tract viral shedding. Of note, chest CT scans may show ground glass opacities even when conventional chest radiographs are unremarkable, improving the sensitivity of diagnosis. Ganciclovir or foscarnet are commonly prescribed for systemic CMV infections. In transplant recipients with CMV pneumonitis, IVIG immune modulation along with effective antiviral drug if recommended. A detailed discussion is provided regarding specific pathogens and approach towards therapeutic management of lung infections in chapters throughout this book.

\section{References}

1. Fishman JA. Infection in solid-organ transplant recipients. N Engl J Med. 2007;357:2601-14.

2. Ibrahim EH, Tracy L, Hill C, Fraser VJ, Kollef MH. The occurrence of ventilator-associated pneumonia in a community hospital: risk factors and clinical outcomes. Chest. 2001;120:555-61.

3. Bodey GP, Buckley M, Sathe YS, Freireich EJ. Quantitative relationships between circulating leukocytes and infection in patients with acute leukemia. Ann Intern Med. 1966;64:328-40.

4. Carlisle PS, Gucalp R, Wiernik PH. Nosocomial infections in neutropenic cancer patients. Infect Control Hosp Epidemiol. 1993;14:320-4.

5. Issa NC, Fishman JA. Infectious complications of antilymphocyte therapies in solid organ transplantation. Clin Infect Dis Off Publ Infect Dis Soc Am. 2009;48:772-86.

6. Roemer E, Blau IW, Basara N, Kiehl MG, Bischoff M, Günzelmann $\mathrm{S}$, et al. Toxoplasmosis, a severe complication in allogeneic hematopoietic stem cell transplantation: successful treatment strategies during a 5-year single-center experience. Clin Infect Dis Off Publ Infect Dis Soc Am. 2001;32:E1-8.

7. Safdar A, Armstrong D. Listeriosis in patients at a comprehensive cancer center, 1955-1997. Clin Infect Dis Off Publ Infect Dis Soc Am. 2003;37:359-64.

8. Savage DG, Taylor P, Blackwell J, Chen F, Szydlo RM, Rule SA, Spencer A, et al. Paranasal sinusitis following allogeneic bone marrow transplant. Bone Marrow Transplant. 1997;19(1):55-9.

9. Shibuya TY, Momin F, Abella E, Jacobs JR, Karanes C, Ratanatharathorn $\mathrm{V}$, et al. Sinus disease in the bone marrow transplant population: incidence, risk factors, and complications. Otolaryngol Head Neck Surg. 1995;113(6):705-11.

10. Dhong HJ, Lee JC, Ryu JS, Cho DY. Rhinosinusitis in transplant patients. Clin Otolaryngol Allied Sci. 2001;26:329-33.

11. Yung MW, Gould J, Upton GJG. Nasal polyposis in children with cystic fibrosis: a long-term follow-up study. Ann Otol Rhinol Laryngol. 2002;111:1081-6.

12. Ortiz E, Sakano E, De Souza CA, Vigorito A, Eid KAB, Chronic GVHD. Predictive factor for rhinosinusitis in bone marrow transplantation. Braz J Otorhinolaryngol. 2006;72:328-32.
13. Thompson AM, Couch M, Zahurak ML, Johnson C, Vogelsang GB. Risk factors for post-stem cell transplant sinusitis. Bone Marrow Transplant. 2002;29:257-61.

14. Rayes A, Sahni K, Hanna C, Suryadevara M, Goyal P, Cherrick I. Cytomegalovirus sinusitis in a child with chronic myelogenous leukemia following bone marrow transplantation. Pediatr Blood Cancer. 2011;56:1140-2.

15. Gwaltney JM. Acute community-acquired sinusitis. Clin Infect Dis. 1996;23(6):1209-23; quiz 1224-5.

16. Mogensen C, Tos M. Quantitative histology of the maxillary sinus. Rhinology. 1977;15:129-40.

17. Fokkens W, Lund V, Mullol J, European Position Paper on Rhinosinusitis and Nasal Polyps Group. EP3OS 2007: European position paper on rhinosinusitis and nasal polyps 2007. A summary for otorhinolaryngologists. Rhinology. 2007;45: 97-101.

18. Holzmann D, Speich R, Kaufmann T, Laube I, Russi EW, Simmen $\mathrm{D}$, et al. Effects of sinus surgery in patients with cystic fibrosis after lung transplantation: a 10-year experience. Transplantation. 2004;77:134-6.

19. Murray TS, Egan M, Kazmierczak BI. Pseudomonas aeruginosa chronic colonization in cystic fibrosis patients. Curr Opin Pediatr. 2007; $19: 83-8$

20. Evans FO, Sydnor JB, Moore WEC, Moore GR, Manwaring JL, Brill AH, et al. Sinusitis of the maxillary antrum. N Engl J Med. 1975;293:735-9.

21. de Medeiros CR, Bleggi-Torres LF, Faoro LN, Reis-Filho JS, Silva LC, de Medeiros BC, et al. Cavernous sinus thrombosis caused by zygomycosis after unrelated bone marrow transplantation. Transpl Infect Dis. 2001;3:231-4.

22. Li JY, Yong TY, Khoo E, Russ GR, Grove DI, Coates PT, McDonald SP. Isolated sphenoid fungal sinusitis in a renal transplant recipient presenting with bilateral abducens nerve palsy. Transpl Int. 2007;20(7):640-2.

23. Berlinger NT. Sinusitis in immunodeficient and immunosuppressed patients. Laryngoscope. 1985;95:29-33.

24. Lee SY, Yeo CL, Lee WH, Kwa AL, Koh LP, Hsu LY. Prevalence of invasive fungal disease in hematological patients at a tertiary university hospital in Singapore. BMC Res Notes. 2011;4:42.

25. Ellison MD, Hung RT, Harris K, Campbell BH. Report of the first case of invasive fungal sinusitis caused by Scopulariopsis acremonium: review of Scopulariopsis infections. Arch Otolaryngol Head Neck Surg. 1998;124:1014-6.

26. Rombaux P, Eloy P, Bertrand B, Delos M, Doyen C. Lethal disseminated Fusarium infection with sinus involvement in the immunocompromised host: case report and review of the literature. Rhinology. 1996;34:237-41.

27. Richardson SE, Bannatyne RM, Summerbell RC, Milliken J, Gold R, Weitzman SS. Disseminated fusarial infection in the immunocompromised host. Rev Infect Dis. 1988;10:1171-81.

28. Furukawa H, Kusne S, Sutton DA, Manez R, Carrau R, Nichols L, et al. Acute invasive sinusitis due to Trichoderma longibrachiatum in a liver and small bowel transplant recipient. Clin Infect Dis. 1998;26:487-9.

29. Machado CM, Martins MA, Heins-Vaccari EM, CdS L, MCA M, Castelli JB, et al. Scedosporium apiospermum sinusitis after bone marrow transplantation: report of a case. Rev Inst Med Trop Sao Paulo. 1998;40:321-3.

30. Castiglioni B, Sutton DA, Rinaldi MG, Fung J, Kusne S. Pseudallescheria boydii (Anamorph Scedosporium apiospermum). Infection in solid organ transplant recipients in a tertiary medical center and review of the literature. Medicine (Baltimore). 2002;81:333-48.

31. Vernon SE, Acar BC, Pham SM, Fertel D. Acanthamoeba infection in lung transplantation: report of a case and review of the literature. Transpl Infect Dis. 2005;7:154-7. 
32. Rivera MA, Padhya TA. Acanthamoeba: a rare primary cause of rhinosinusitis. Laryngoscope. 2002;112:1201-3.

33. Craven DE, Hjalmarson KI. Ventilator-associated tracheobronchitis and pneumonia: thinking outside the box. Clin Infect Dis. 2010;51(Suppl 1):S59-66.

34. van Assen S, Bootsma GP, Verweij PE, Donnelly JP, Raemakers JM. Aspergillus tracheobronchitis after allogeneic bone marrow transplantation. Bone Marrow Transplant. 2000;26:1131-2.

35. Fernández-Ruiz M, Silva JT, San-Juan R, de Dios B, García-Luján R, López-Medrano F, et al. Aspergillus tracheobronchitis: report of 8 cases and review of the literature. Medicine (Baltimore). 2012;91:261-73.

36. Wenzel RP, Fowler AA. Clinical practice. Acute bronchitis. N Engl J Med. 2006;355:2125-30.

37. Falsey AR, Erdman D, Anderson LJ, Walsh EE. Human metapneumovirus infections in young and elderly adults. J Infect Dis. 2003;187:785-90.

38. Boldy DA, Skidmore SJ, Ayres JG. Acute bronchitis in the community: clinical features, infective factors, changes in pulmonary function and bronchial reactivity to histamine. Respir Med. 1990;84:377-85.

39. Jonsson JS, Sigurdsson JA, Kristinsson KG, Guthnadóttir M, Magnusson S. Acute bronchitis in adults. How close do we come to its aetiology in general practice? Scand J Prim Health Care. 1997; 15:156-60.

40. Louie JK, Hacker JK, Gonzales R, Mark J, Maselli JH, Yagi $\mathrm{S}$, Drew WL. Characterization of viral agents causing acute respiratory infection in a San Francisco University Medical Center Clinic during the influenza season. Clin Infect Dis. 2005;41:822-8.

41. Gottlieb J, Schulz TF, Welte T, Fuehner T, Dierich M, Simon AR, Engelmann I. Community-acquired respiratory viral infections in lung transplant recipients: a single season cohort study. Transplantation. 2009;87:1530-7.

42. Chemaly RF, Ghosh S, Bodey GP, Rohatgi N, Safdar A, Keating MJ, et al. Respiratory viral infections in adults with hematologic malignancies and human stem cell transplantation recipients: a retrospective study at a major cancer center. Medicine (Baltimore). 2006;85:278-87.

43. Renaud C, Campbell AP. Changing epidemiology of respiratory viral infections in hematopoietic cell transplant recipients and solid organ transplant recipients. Curr Opin Infect Dis. 2011;24:333-43.

44. Tang T-C, Chuang W-Y. Aspergillus tracheobronchitis in allogeneic stem cell transplanted recipient. Eur J Haematol. 2012;88:183.

45. Krenke R, Kołkowska-Leśniak A, Pałynyczko G, ProchorecSobieszek M, Konopka L. Ulcerative and pseudomembranous Aspergillus tracheobronchitis in a patient with acute myeloid leukemia. Int J Hematol. 2009;89:257-8.

46. Adams PF, Hendershot GE, Marano MA, Centers for Disease Control and Prevention/National Center for Health Statistics. Current estimates from the National Health Interview Survey, 1996. Vital Health Stat. 1999;10:1-203.

47. Ljungman P, Ward KN, Crooks BN, Parker A, Martino R, Shaw PJ, et al. Respiratory virus infections after stem cell transplantation: a prospective study from the Infectious Diseases Working Party of the European Group for Blood and Marrow Transplantation. Bone Marrow Transplant. 2001;28:479-84.

48. Kumar D, Husain S, Chen MH, Moussa G, Himsworth D, Manuel $\mathrm{O}$, et al. A prospective molecular surveillance study evaluating the clinical impact of community-acquired respiratory viruses in lung transplant recipients. Transplantation. 2010;89:1028-33.

49. López-Medrano F, Aguado JM, Lizasoain M, Folgueira D, Juan RS, Díaz-Pedroche C, et al. Clinical implications of respiratory virus infections in solid organ transplant recipients: a prospective study. Transplantation. 2007;84:851-6.
50. Erard V, Chien JW, Kim HW, Nichols WG, Flowers ME, Martin PJ, et al. Airflow decline after myeloablative allogeneic hematopoietic cell transplantation: the role of community respiratory viruses. J Infect Dis. 2006;193:1619-25.

51. Waghmare A, Englund JA, Boeckh M. How I treat respiratory viral infections in the setting of intensive chemotherapy or hematopoietic cell transplantation. Blood. 2016;127:2682-92.

52. Campbell AP, Guthrie KA, Englund JA, Farney RM, Minerich EL, Kuypers J, et al. Clinical outcomes associated with respiratory virus detection before allogeneic hematopoietic stem cell transplant. Clin Infect Dis. 2015;61:192-202.

53. Fisher CE, Preiksaitis CM, Lease ED, Edelman J, Kirby KA, Leisenring WM, et al. Symptomatic respiratory virus infection and chronic lung allograft dysfunction. Clin Infect Dis. 2016;62:313-9.

54. Chien JW, Duncan S, Williams KM, Pavletic SZ. Bronchiolitis obliterans syndrome after allogeneic hematopoietic stem cell transplantation-an increasingly recognized manifestation of chronic graft-versus-host disease. Biol Blood Marrow Transplant. 2010;16:S106-14.

55. Mandell LA, Wunderink RG, Anzueto A, Bartlett JG, Campbell GD, Dean NC, Infectious Diseases Society of America; American Thoracic Society, et al. Infectious Diseases Society of America/ American Thoracic Society consensus guidelines on the management of community-acquired pneumonia in adults. Clin Infect Dis. 2007;44:S27-72.

56. Kalil AC, Metersky ML, Klompas M, Muscedere J, Sweeney DA, Palmer LB, et al. Management of adults with hospital-acquired and ventilator-associated pneumonia: 2016 clinical practice guidelines by the Infectious Diseases Society of America and the American Thoracic Society. Clin Infect Dis. 2016;63(5):e61-e111.

57. Costa SF, Alexander BD. Non-Aspergillus fungal pneumonia in transplant recipients. Clin Chest Med. 2005;26:675-90, vii.

58. Marr KA, Carter RA, Crippa F, Wald A, Corey L. Epidemiology and outcome of mould infections in hematopoietic stem cell transplant recipients. Clin Infect Dis. 2002;34:909-17.

59. Kotloff RM, Ahya VN, Crawford SW. Pulmonary complications of solid organ and hematopoietic stem cell transplantation. Am J Respir Crit Care Med. 2004;170:22-48.

60. Chalmers JD, Rother C, Salih W, Ewig S. Healthcare-associated pneumonia does not accurately identify potentially resistant pathogens: a systematic review and meta-analysis. Clin Infect Dis. 2014;58:330-9.

61. Gross AE, Van Schooneveld TC, Olsen KM, Rupp ME, Bui TH, Forsung E, Kalil AC. Epidemiology and predictors of multidrugresistant community-acquired and health care-associated pneumonia. Antimicrob Agents Chemother. 2014;58:5262-8.

62. Yap V, Datta D, Metersky ML. Is the present definition of health care-associated pneumonia the best way to define risk of infection with antibiotic-resistant pathogens? Infect Dis Clin N Am. 2013;27:1-18.

63. Bartlett JG. Community-acquired pneumonia. Int J Pract Suppl. 2000;115:18-22.

64. Aisenberg G, Rolston KV, Safdar A. Bacteremia caused by Achromobacter and Alcaligenes species in 46 patients with cancer (1989-2003). Cancer. 2004;101:2134-40.

65. Patterson TF, Kirkpatrick WR, White M, Hiemenz JW, Wingard JR, Dupont B, et al. Invasive aspergillosis. Disease spectrum, treatment practices, and outcomes. I3 Aspergillus Study Group. Medicine (Baltimore). 2000;79:250-60.

66. Junghanss C, Marr KA, Carter RA, Sandmaier BM, Maris MB, Maloney DG, et al. Incidence and outcome of bacterial and fungal infections following nonmyeloablative compared with myeloablative allogeneic hematopoietic stem cell transplantation: a matched control study. Biol Blood Marrow Transplant. 2002;8:512-20. 
67. Marr KA, Carter RA, Boeckh M, Martin P, Corey L. Invasive aspergillosis in allogeneic stem cell transplant recipients: changes in epidemiology and risk factors. Blood. 2002;100:4358-66.

68. Ison MG, Hayden FG, Kaiser L, Corey L, Boeckh M. Rhinovirus infections in hematopoietic stem cell transplant recipients with pneumonia. Clin Infect Dis. 2003;36:1139-43.

69. Alangaden GJ, Wahiduzzaman M, Chandrasekar PH, Bone Marrow Transplant Group. Aspergillosis: the most common community-acquired pneumonia with gram-negative Bacilli as copathogens in stem cell transplant recipients with graft-versushost disease. Clin Infect Dis. 2002;35:659-64.

70. Ascioglu S, Rex JH, de Pauw B, Bennett JE, Bille J, Crokaert F, Invasive Fungal Infections Cooperative Group of the European Organization for Research and Treatment of Cancer, Mycoses Study Group of the National Institute of Allergy and Infectious Diseases, et al. Defining opportunistic invasive fungal infections in immunocompromised patients with cancer and hematopoietic stem cell transplants: an international consensus. Clin Infect Dis. 2002;34:7-14.

71. Mandell LA, Bartlett JG, Dowell SF, File TM Jr, Musher DM, Whitney C, Infectious Diseases Society of America. Update of practice guidelines for the management of community-acquired pneumonia in immunocompetent adults. Clin Infect Dis. 2003;37:1405-33.

72. Niederman MS, Mandell LA, Anzueto A, Bass JB, Broughton WA, Campbell GD, American Thoracic Society, et al. Guidelines for the management of adults with community-acquired pneumonia. Diagnosis, assessment of severity, antimicrobial therapy, and prevention. Am J Respir Crit Care Med. 2001;163:1730-54.

73. Rubinstein E, Kollef MH, Nathwani D. Pneumonia caused by methicillin-resistant Staphylococcus aureus. Clin Infect Dis. 2008;46:S378-85.

74. Rello J, Ollendorf DA, Oster G, Vera-Llonch M, Bellm L, Redman R, Kollef MH, VAP Outcomes Scientific Advisory Group. Epidemiology and outcomes of ventilator-associated pneumonia in a large US database. Chest. 2002;122:2115-21.

75. American Thoracic Society \& Infectious Diseases Society of America. Guidelines for the management of adults with hospitalacquired, ventilator-associated, and healthcare-associated pneumonia. Am J Respir Crit Care Med. 2005;171:388-416.

76. Dangerfield B, Chung A, Webb B, Seville MT. Predictive value of methicillin-resistant Staphylococcus aureus (MRSA) nasal swab PCR assay for MRSA pneumonia. Antimicrob Agents Chemother. 2014;58:859-64.

77. Reza Shariatzadeh M, Huang JQ, Marrie TJ. Differences in the features of aspiration pneumonia according to site of acquisition: community or continuing care facility. J Am Geriatr Soc. 2006;54:296-302.

78. Marik PE. Aspiration pneumonitis and aspiration pneumonia. $\mathrm{N}$ Engl J Med. 2001;344:665-71.

79. Cabre M, Serra-Prat M, Palomera E, Almirall J, Pallares R, Clavé P. Prevalence and prognostic implications of dysphagia in elderly patients with pneumonia. Age Ageing. 2010;39:39-45.

80. El-Solh AA, Pietrantoni C, Bhat A, Aquilina AT, Okada M, Grover V, Gifford N. Microbiology of severe aspiration pneumonia in institutionalized elderly. Am J Respir Crit Care Med. 2003; 167:1650-4.

81. Mier L, Dreyfuss D, Darchy B, Lanore JJ, Djedaïni K, Weber $\mathrm{P}$, et al. Is penicillin $\mathrm{G}$ an adequate initial treatment for aspiration pneumonia? A prospective evaluation using a protected specimen brush and quantitative cultures. Intensive Care Med. 1993;19:279-84.

82. Carratalà J, Mykietiuk A, Fernández-Sabé N, Suárez C, Dorca J, Verdaguer R, et al. Health care-associated pneumonia requiring hospital admission: epidemiology, antibiotic therapy, and clinical outcomes. Arch Intern Med. 2007;167:1393-9.
83. Brown-Elliott BA, Brown JM, Conville PS, Wallace RJ. Clinical and laboratory features of the Nocardia spp. based on current molecular taxonomy. Clin Microbiol Rev. 2006;19:259-82.

84. Torres HA, Reddy BT, Raad II, Tarrand J, Bodey GP, Hanna HA, et al. Nocardiosis in cancer patients. Medicine (Baltimore). 2002;81:388-97.

85. Peleg AY, Husain S, Qureshi ZA, Silveira FP, Sarumi M, Shutt KA, et al. Risk factors, clinical characteristics, and outcome of Nocardia infection in organ transplant recipients: a matched casecontrol study. Clin Infect Dis. 2007;44:1307-14.

86. Santos M, Gil-Brusola A, Morales P. Infection by Nocardia in solid organ transplantation: thirty years of experience. Transplant Proc. 2011;43:2141-4.

87. Yu X, Han F, Wu J, He Q, Peng W, Wang Y, et al. Nocardia infection in kidney transplant recipients: case report and analysis of 66 published cases. Transpl Infect Dis. 2011;13:385-91.

88. Uttamchandani RB, Daikos GL, Reyes RR, Fischl MA, Dickinson GM, et al. Nocardiosis in 30 patients with advanced human immunodeficiency virus infection: clinical features and outcome. Clin Infect Dis. 1994;18:348-53.

89. De La Rosa GR, Jacobson KL, Rolston KV, Raad II, Kontoyiannis DP, Safdar A. Mycobacterium tuberculosis at a comprehensive cancer centre: active disease in patients with underlying malignancy during 1990-2000. Clin Microbiol Infect. 2004;10:749-52.

90. Muñoz P, Rodríguez C, Bouza E. Mycobacterium tuberculosis infection in recipients of solid organ transplants. Clin Infect Dis. 2005;40:581-7.

91. Singh N, Paterson DL. Mycobacterium tuberculosis infection in solid-organ transplant recipients: impact and implications for management. Clin Infect Dis. 1998;27:1266-77.

92. Keating MR, Daly JS, AST Infectious Diseases Community of Practice. Nontuberculous mycobacterial infections in solid organ transplantation. Am J Transplant. 2013;13(Suppl 4):77-82.

93. Patel R, Roberts GD, Keating MR, Paya CV. Infections due to nontuberculous mycobacteria in kidney, heart, and liver transplant recipients. Clin Infect Dis. 1994;19:263-73.

94. Knoll BM, Kappagoda S, Gill RR, Goldberg HJ, Boyle K, Baden LR, et al. Non-tuberculous mycobacterial infection among lung transplant recipients: a 15 -year cohort study. Transpl Infect Dis. 2012;14:452-60.

95. Piersimoni C. Nontuberculous mycobacteria infection in solid organ transplant recipients. Eur J Clin Microbiol Infect Dis. 2012;31:397-403.

96. Safdar A, White DA, Stover D, Armstrong D, Murray HW. Profound interferon gamma deficiency in patients with chronic pulmonary nontuberculous mycobacteriosis. Am J Med. 2002;113:756-9.

97. Martin SI, Fishman JA, AST Infectious Diseases Community of Practice. Pneumocystis pneumonia in solid organ transplantation. Am J Transplant. 2013;13(Suppl 4):272-9.

98. Morgan J, Wannemuehler KA, Marr KA, Hadley S, Kontoyiannis DP, Walsh TJ, et al. Incidence of invasive aspergillosis following hematopoietic stem cell and solid organ transplantation: interim results of a prospective multicenter surveillance program. Med Mycol. 2005;43(Suppl 1):S49-58.

99. Marr KA. Empirical antifungal therapy - new options, new tradeoffs. N Engl J Med. 2002;346:278-80.

100. Marr KA, Patterson T, Denning D. Aspergillosis. Pathogenesis, clinical manifestations, and therapy. Infect Dis Clin N Am. 2002;16:875-94, vi.

101. Singh N, Singh NM, Husain S, AST Infectious Diseases Community of Practice. Aspergillosis in solid organ transplantation. Am J Transplant. 2013;13(Suppl 4):228-41.

102. Kontoyiannis DP, Lionakis MS, Lewis RE, Chamilos G, Healy M, Perego $\mathrm{C}$, et al. Zygomycosis in a tertiary-care cancer center in the 
era of Aspergillus-active antifungal therapy: a case-control observational study of 27 recent cases. J Infect Dis. 2005;191:1350-60.

103. Heussel CP, Kauczor HU, Heussel GE, Fischer B, Begrich M, Mildenberger P, Thelen M. Pneumonia in febrile neutropenic patients and in bone marrow and blood stem-cell transplant recipients: use of high-resolution computed tomography. J Clin Oncol. 1999; 17:796-805.

104. Razonable R. Direct and indirect effects of Cytomegalovirus: can we prevent them? Enferm Infecc Microbiol Clin. 2010;28:1-5.
105. George MJ, Snydman DR, Werner BG, Griffith J, Falagas ME, Dougherty NN, Rubin RH. The independent role of Cytomegalovirus as a risk factor for invasive fungal disease in orthotopic liver transplant recipients. Boston Center for Liver Transplantation CMVIG-Study Group. Cytogam, MedImmune, Inc. Gaithersburg, Maryland. Am J Med. 1997;103:106-13.

106. Walker RC, Marshall WF, Strickler JG, Wiesner RH, Velosa JA, Habermann TM, et al. Pretransplantation assessment of the risk of lymphoproliferative disorder. Clin Infect Dis. 1995;20:1346-53. 